\title{
IMPACT OF THE INSERTABLE B-LAYER ON $b$-TAGGING PERFORMANCE FOR ATLAS RUN-II*
}

\author{
Sylvestre Pires \\ on behalf of the ATLAS Collaboration
}

Laboratoire de Physique Nucléaire et de Hautes Énergies, UPMC

Université Paris-Diderot, CNRS/IN2P3 — Paris, France

(Received May 7, 2014)

The $b$-tagging performance expected for the ATLAS experiment at the Run-II of the Large Hadron Collider (LHC) will be presented hereafter. These studies are based on Monte Carlo simulations of $t \bar{t}$ events. These results allow to quantify the impact on the $b$-tagging performance of both the installation of a new pixel layer and the repairs of several electronics components of the present Pixel Detector. Beforehand, a brief description of the ATLAS experiment at the LHC and of the $b$-tagging algorithms will be given.

DOI:10.5506/APhysPolB.45.1567

PACS numbers: 07.05.Fb, 07.05.Hd, 14.64.Fy, 29.40.Gx

\section{Introduction}

\subsection{The $L H C$ and the ATLAS detector}

In 2015 the Large Hadron Collider (LHC) [1] will upgrade its energy to 13-14 TeV and its peak luminosity to $\mathcal{L}=10^{34} \mathrm{~cm}^{-2} \mathrm{~s}^{-1}$, planning to reach $\mathcal{L}=2 \times 10^{34} \mathrm{~cm}^{-2} \mathrm{~s}^{-1}$ in 2020. The ATLAS (A Toroidal LHC ApparatuS) detector [2] consists of an Inner Detector (ID) tracking system surrounded by a superconducting solenoid providing a $2 \mathrm{~T}$ magnetic field, electromagnetic and hadronic calorimeters, and a muon spectrometer (MS). Among the ID sub-detectors, the closest to the beam axis consists of pixel and silicon microstrip detectors inside a transition radiation tracker. The electromagnetic calorimeter is a lead liquid-argon ( $\mathrm{LAr}$ ) detector with a barrel covering $|\eta|<1.475$ and endcap regions at $1.375<|\eta|<3.2$. Hadron calorimetry is based on two different detector technologies. The barrel $(|\eta|<0.8)$

* Presented at the Cracow Epiphany Conference on the Physics at the LHC, Kraków, Poland, January 8-10, 2014. 
and extended barrel $(0.8<|\eta|<1.7)$ calorimeters are composed of scintillator/steel, while the hadronic endcap calorimeters $(1.5<|\eta|<3.2)$ are LAr/copper. The forward calorimeters $(3.1<|\eta|<4.9)$ are instrumented with LAr/tungsten and LAr/copper, providing electromagnetic and hadronic energy measurements, respectively. The Muon Spectrometer consists of three large superconducting toroids and a system of three stations of trigger chambers and precision tracking chambers. A three-level trigger and dataflow system enables an event selection with a rate of about $300 \mathrm{~Hz}$.

\subsection{Motivation for the $I B L$}

The ID consists of three sub-detectors. The closest to the beams is the Pixel Detector [3] composed of three concentric layers of sensors assembled into 1744 modules with 80 millions of pixels. Before 2020, ATLAS is expected to collect $300 \mathrm{fb}^{-1}$ of data, for which the expected fluence of $50 \mathrm{MRad}$ ionising dose and $10^{15} \mathrm{n}_{\text {eq }} / \mathrm{cm}^{2}$ non-ionising dose will lead to significant radiation damage to the Pixel Detector [4]. To mitigate these effects, a new fourth layer of pixel modules, the Insertable B-Layer (IBL), will be inserted between the current Pixel Detector and a new smaller radius beam-pipe [4] (cf. Fig. 1). The IBL sensors will be located at an average radius of $3.3 \mathrm{~cm}$ from the beam-axis and cover the pseudorapidity range $|\eta|<2.9$. Due to the close proximity to the interaction point, the IBL must withstand $250 \mathrm{MRad}$ of ionising dose and $5 \times 10^{15} \mathrm{n}_{\text {eq }} / \mathrm{cm}^{2}$ non-ionising dose over the Run-II.

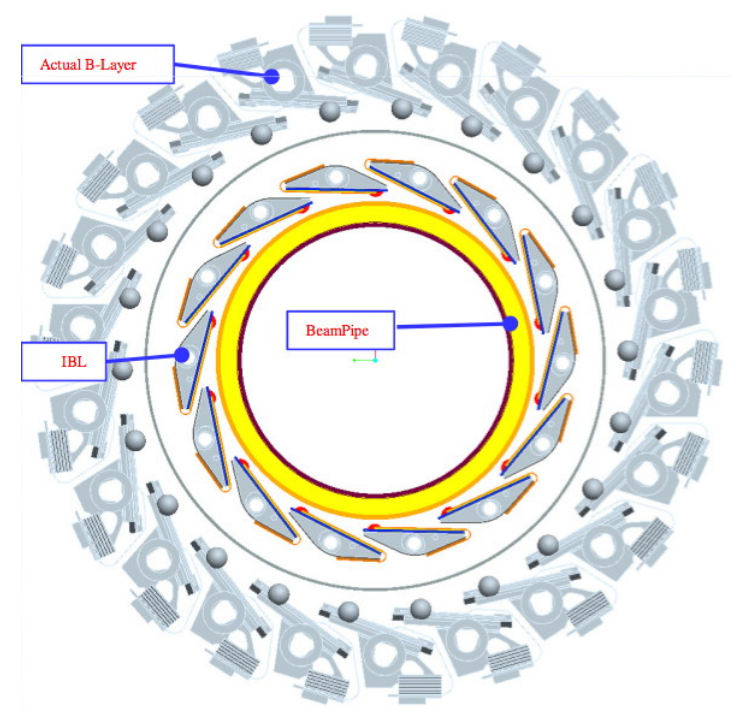

Fig. 1. Schematic view of the Insertable B-Layer, at $3.3 \mathrm{~cm}$ of the interaction point and the actual B-Layer at $\sim 5 \mathrm{~cm}$ radius [4]. 
The IBL will improve $b$-tagging performance by introducing this $4^{\text {th }}$, high granularity, pixel layer that will provide redundancy of the actual first layer (the B-Layer) measurements and improve the tracking performance thanks to lower pixel occupancy compared to the actual B-Layer.

\section{Tagging of $b$-jets}

The identification of jets stemming from a $b$-quark is important to many physics analyses, including the $H \rightarrow b \bar{b}$ channel, top quark studies (through its exclusive decay mode $t \rightarrow W b$ ) and for various studies beyond the Standard Model.

The $b$-tagging algorithms combine the information from the tracks, the primary vertex and the jet reconstruction. Within the ATLAS Collaboration, the algorithm mainly used for the jets reconstruction is anti- $k_{\mathrm{t}}$ [5]. The $b$-jet tagging techniques take advantage of the relatively long lifetime ( $c \tau \simeq 450 \mu \mathrm{m}$ ) of hadrons containing $b$-quarks, as well as of the hard fragmentation and the high mass of the $b$ hadrons. These properties lead to tracks with large impact parameters with respect to the primary vertex, as illustrated in Fig. 2, and to secondary vertices with large invariant masses.

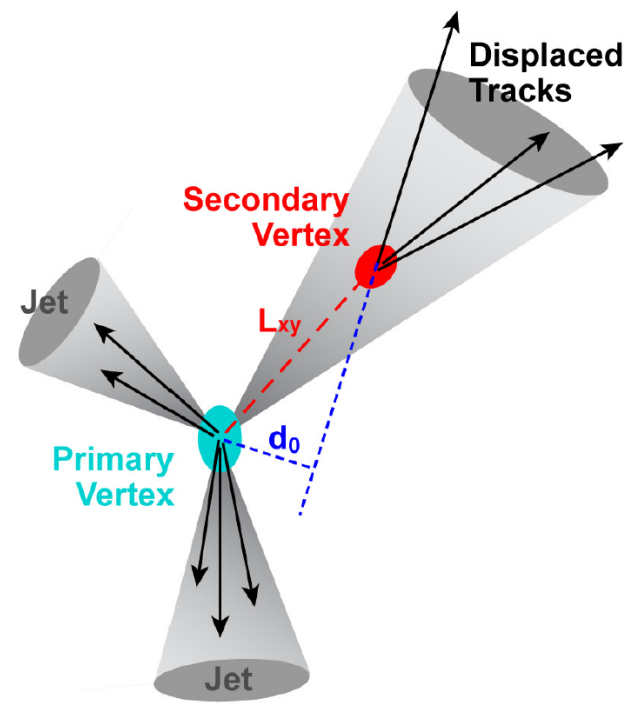

Fig. 2. Schematic view of a jet containing a secondary vertex and displaced tracks, signature of a $b$-jet. Tracks are represented by arrows and the circles mark the primary (grey/blue) and secondary (dark grey/red) vertices. The transverse decay length $L_{x y}$ and the transverse impact parameter distance $d_{0}$ which characterise the secondary vertex are indicated by dashed lines. 
Figure 3 shows the performance of different $b$-tagging algorithms as measured by ATLAS based on simulated $t \bar{t}$ events at $7 \mathrm{TeV}$ [6], plotted in terms of the light jet rejection factors as a function of the $b$-tagging efficiency. The simplest algorithm (named SV0) is based on the reconstruction of the distance of the transverse decay length $L_{x y}$ of the $b$ hadron, which is the vector pointing from the primary vertex to the $b$ hadron decay vertex. The tracking information also allows to reconstruct the transverse impact parameter $d_{0}$, which is the closest distance of approach of the track to the collision point. A second method (IP2D) to tag the $b$-jets is based on the use of the signed impact parameter. Several more complex algorithms have been developed by the ATLAS Collaboration. Among them, two will be our references in the following, IP3D and SV1 [7]. The first one is based on the combination of the transverse $\left(d_{0} / \sigma\left(d_{0}\right)\right)$ and longitudinal $\left(z_{0} / \sigma\left(z_{0}\right)\right)$ impact parameters significance. The second one is built on four properties of the secondary vertex: the invariant mass of all the tracks associated to the vertex, the ratio of the energies sum of the tracks in the vertex to the energies sum of all tracks in the jet, the number of two-track vertices in the jet, and the distance $\Delta R$ between the jet axis and the line joining the primary to the secondary vertex. These two algorithms are then combined by a likelihood ratio. As can be seen in Fig. 3, the combination SV1+IP3D keeps one light

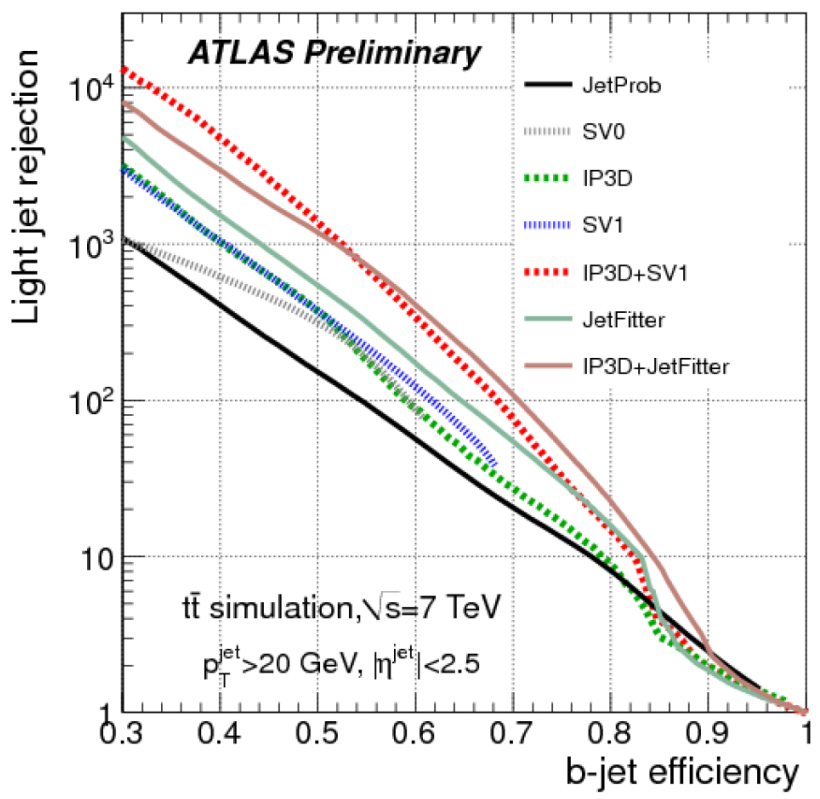

Fig. 3. Light jet rejection as a function of the $b$-tagging efficiency for different algorithms based on simulated $t \bar{t}$ events at $7 \mathrm{TeV}[6]$. 
jet over 100 for a $b$-tagging efficiency of $70 \%$. Other algorithms, based on multivariate methods, give higher performance, but are not yet considered for these studies.

\section{Results obtained}

The impact of the IBL on $b$-tagging performance has been studied in the technical design report (TDR) of IBL [4]. Since then, more refined detector description, reconstruction techniques and more realistic conditions of data taking have been studied.

\subsection{Impact of the IBL installation}

Figure 4 shows the rejection factor for light jets as a function of the efficiency for $b$-jets, measured by varying the cut on the jet weight obtained from the combination of the taggers IP3D $+\mathrm{SV} 1$. The results are obtained using $t \bar{t}$ simulated events without pileup, where pileup is defined as the number of interactions per bunch crossing.

Jets used for this study have $p_{\mathrm{T}}>15 \mathrm{GeV},|\eta|<2.5$ and at least one track associated to the vertex. Including the IBL in the ATLAS geometry leads to a significant improvement in the rejection of light jets at fixed $b$ efficiency.

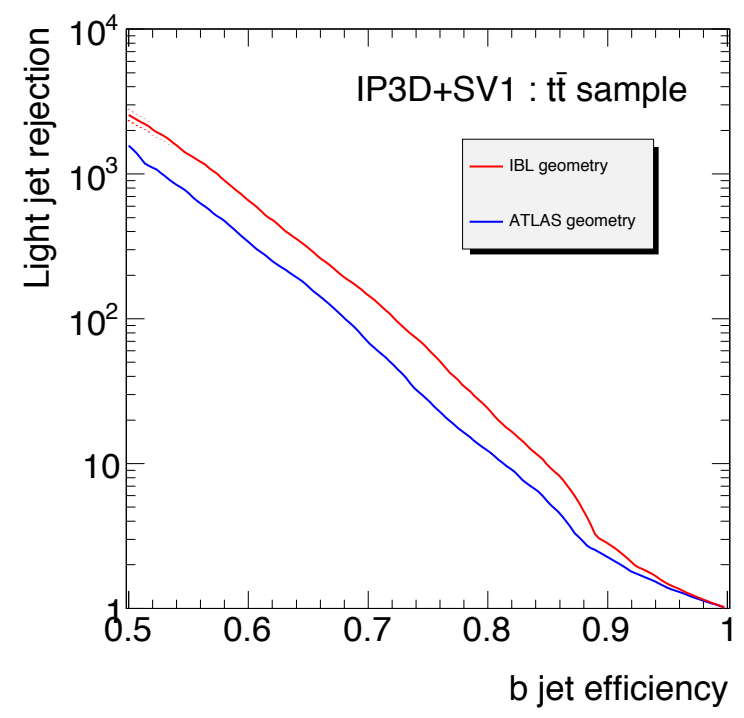

Fig. 4. Rejection factor against light jets as a function of $b$-jet efficiency for the IP3D + SV1 tagger. Compared are the results with and without IBL, using $b$ and light jets from $t \bar{t}$ simulated events without pileup [4]. 


\subsection{Performance expected}

The Pixel Detector was subject to a very high radiation dose all along ATLAS Run-I. Along with time and the luminosity increase, damages have been observed, among others, on the opto-boards which enable pixels connection to the electronics systems and other connections services, namely the Service Quarter Panels (SQP). Several scenarii of modules failures with time have been developed. At the end of Run-I, a total of 88 modules in the Pixel Detector have failed, with about $2 / 3$ of them due to opto-boards failures. The dead modules are currently being repaired, with the installation of new opto-board and new service quarter panels (nSQP), therefore, there will be only around 20 dead modules at the start of Run-II. Figure 5 shows the number of disabled modules observed and expected in the Pixel Detector, along with time. From linear extrapolation of module failure rate, it is expected to have around $44(2 \%)$ dead modules in 2019.

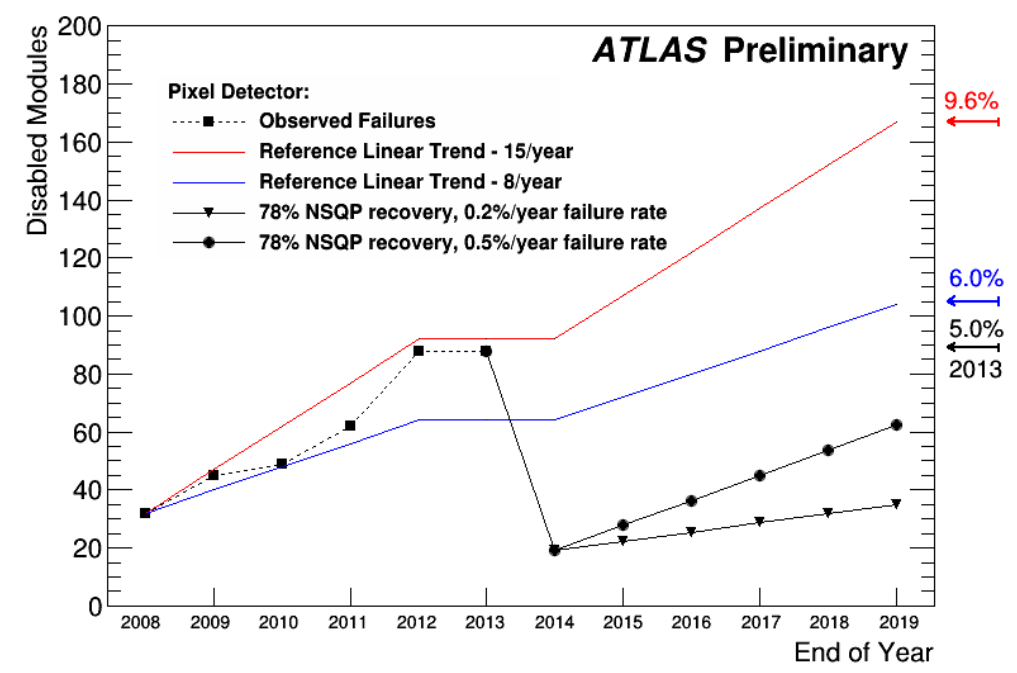

Fig. 5. Number of disabled modules observed and expected in the Pixel Detector, along with time [8].

Four different geometry scenarii have been developed, aiming to study the $b$-tagging performance with IBL in 2019:

1. No IBL module disabled in 2019 and 35 disabled modules in the Pixel Detector, corresponding to the number of repaired modules in 2014.

2. 5 IBL modules $(\sim 2 \%)$ failures and 35 disabled modules in the Pixel Detector. 
3. 5 IBL modules failures and 167 disabled modules in the Pixel Detector, corresponding to a scenario without module repairs.

4. 5 IBL modules failures and 167 disabled modules in the Pixel Detector, with $50 \%$ of the events going through an additional reading limitation, corresponding to the first layer of the Pixel Detector totally disabled.

Figure 6 shows the rejection factor against light jets as a function of the $b$-jet efficiency for the 4 scenarii described above. The results are obtained with $t \bar{t}$ events simulation assuming the pileup of 60 , as expected throughout Run-II. This study has been conducted in order to understand the effects of modules repairs on the $b$-tagging performance. The modules repairs improves the light jet rejection by $\sim 20 \%$ at fixed $b$-tagging efficiency. This study also allows us to estimate the impact of the IBL modules failures on $b$-tagging performance. In scenario 1 , assuming a fully operational IBL and Pixel Detector modules repairs, the light jet rejection would be $10 \%$ better than in scenario 2 , where $2 \%$ of the IBL modules have failed throughout Run-II. Finally, it seems that even in the worst case scenario of module degradation, scenario 4 , the loss of $b$-tagging performance is lower than $40 \%$.

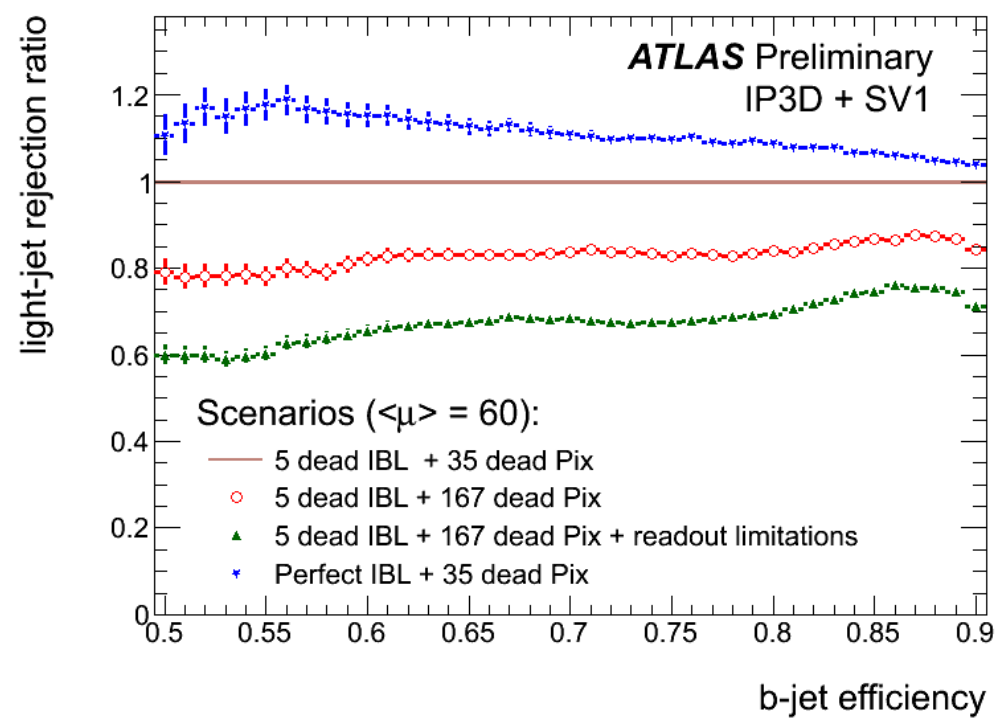

Fig. 6. Rejection factor normalized to 5 dead IBL +35 dead Pix against light jets as a function of $b$-jet efficiency, for the 4 scenarii [8]. 


\section{Conclusion}

The studies made on simulated data allow to evaluate the $b$-tagging performance expected throughout ATLAS Run-II. The IBL installation and the repairing of the Pixel Detector modules failed during ATLAS Run-I will permit to maintain good performance until the end of Run-II. The ongoing optimisation of the ATLAS Collaboration software, taking into account IBL specificities, will further improve the $b$-tagging performance.

This work has been supported by the Polish-French collaboration IN2P3COPIN-40 between IFJ-PAN Kraków and LPHNE Paris.

\section{REFERENCES}

[1] L. Evans, P. Bryant, JINST 3, S08001 (2008).

[2] ATLAS Collaboration, JINST 3, S08003 (2008).

[3] ATLAS Collaboration, JINST 3, P07007 (2008).

[4] ATLAS Collaboration, ATLAS Insertable B-Layer, Technical Design Report, CERN-LHCC-2010-013, ATLAS-TDR-019, 2010.

[5] M. Cacciari, G.P. Salam, G. Soyez, J. High Energy Phys. 0804, 063 (2008) [arXiv:0802.1189 [hep-ph]].

[6] ATLAS Collaboration, ATLAS-CONF-2012-043, 2012, https://cds.cern.ch/record/1435197

[7] ATLAS Collaboration, ATLAS-CONF-2011-102, 2011, https://cds.cern.ch/record/1369219

[8] ATLAS Collaboration, https://twiki.cern.ch/twiki/bin/view/AtlasPublic/PixelPublicResults; https://twiki.cern.ch/twiki/bin/view/AtlasPublic/ApprovedPlotsPixel 